\title{
EDUCAÇÃO PERMANENTE COMO ESTRATÉGIA PARA APRIMORAMENTO DE REGISTROS DE ENFERMAGEM
}

\author{
PERMANENT EDUCATION AS A STRATEGY \\ FOR IMPROVING NURSING RECORDS
}

\section{LA EDUCACIÓN PERMANENTE COMO ESTRATEGIA PARA EL MEJORAMIENTO DE LOS REGISTROS DE ENFERMERÍA}

\author{
Neuranides Santana ${ }^{1}$ \\ Maiara da Silva Brandão Rodrigues ${ }^{2}$ \\ Caren Lorena Menezes Freitas ${ }^{2}$ \\ Rebeca Lopes Oliveira ${ }^{2}$ \\ Danielle Silva dos Santos ${ }^{2}$ \\ Luanda Karina Oliveira de Sousa Barbosa ${ }^{2}$
}

Como citar este artigo: Santana N, Rodrigues MSB, Freitas CLM, Oliveira RL, Santos DS, Barbosa LKOS. Educação permanente como estratégia para aprimoramento de registros de enfermagem. Rev baiana enferm. 2019;33:e33378.

\begin{abstract}
Objetivo: relatar experiência de Educação Permanente em Saúde para aprimoramento dos registros de enfermagem em prontuários. Método: estudo qualitativo, descritivo, do tipo relato de experiência, realizado em hospital público de Salvador, Bahia, entre 14 de junho e 19 de julho de 2018. Os registros de enfermagem foram observados em cinco unidades de internação e apresentados em rodas de conversa. Resultados: os profissionais reconheceram a deficiência dos registros e associaram à falta de atenção, sobrecarga de trabalho devido ao subdimensionamento de pessoal, desconhecimento da legalidade, insuficiência de espaço para registro de intervenções realizadas e à escassez de tempo para atender às demandas do setor. Conclusão: a experiência confirmou que a Educação Permanente em Saúde, além de favorecer aprendizagem significativa, pode transformar os processos de trabalho da saúde, por ser uma modalidade de suporte e elemento de apoio para qualificar o trabalho em saúde, neste caso, o aprimoramento dos registros de enfermagem em prontuários.
\end{abstract}

Descritores: Registro de Enfermagem. Educação Permanente. Cuidados de Enfermagem. Processo de Enfermagem. Segurança do Paciente.

Objective: to report the experience of Permanent Health Education for improving nursing records in medical charts. Method: qualitative, descriptive study, of the experience-report type, performed at a public hospital in Salvador, Babia, between 14 June and 19 July 2018. The nursing records were observed at five inpatient units and presented in wheels of conversation. Results: the professionals acknowledged the deficiency of records and associated it with lack of attention, work overload due to the team sub-dimensioning, lack of legality, insufficient space to record interventions and shortage of time to meet the demands of the sector. Conclusion: the experience has confirmed that the Permanent Health Education, in addition to fostering meaningful learning, can transform health work processes, since it is a modality of support and supporting element to qualify the healthcare work, in this case, enhance nursing records in the charts.

Descriptors: Nursing Records. Education, Continuing. Nursing Care. Nursing Process. Patient Safety.

\footnotetext{
Enfermeira. Doutora em Enfermagem. Professora da Universidade Federal da Bahia. Salvador, Bahia, Brasil. https://orcid.org/orcid.org/0000-0003-0553-264X

Estudante de Enfermagem. Universidade Federal da Bahia. Salvador, Bahia, Brasil. caren3_lore5@hotmail.com. https://orcid.org/0000-0003-3269-1953;
} https://orcid.org/0000-000 I-6678-8193; https://orcid.org/0000-000 I-7850-1380; https://orcid.org/0000-000 I-57 | 8-1664; https://orcid.org/0000-0002-6200-8337. 
Objetivo: presentar la experiencia de educación permanente en salud para la mejora de los registros de enfermería en los prontuarios. Método: estudio cualitativo, descriptivo del tipo relato de experiencia, realizado en un hospital público en Salvador, Babia, entre el 14 de junio y 19 de julio de 2018. Los registros de enfermería fueron observados en cinco unidades de hospitalización y presentados en las ruedas de conversación. Resultados: los profesionales reconocieron la deficiencia de registros, asociándola con la falta de atención, la sobrecarga de trabajo debido a la falta de personal, la falta de legalidad, espacio insuficiente para grabar las intervenciones y la escasez de tiempo para satisfacer las demandas del sector. Conclusión: la experiencia confirmó que la educación permanente en salud, además de fomentar el aprendizaje significativo, puede transformar los procesos de trabajo de salud, al ser una modalidad de soporte y elemento de apoyo para calificar la labor en el cuidado de la salud, en este caso, la mejora de los registros de enfermería en los prontuarios.

Descriptores: Registros de Enfermería. Educación Continua. Atención de Enfermería. Proceso de Enfermería. Seguridad del Paciente.

\section{Introdução}

O trabalho em saúde é coletivo e segmentado. Por esse motivo, para dar continuidade ao cuidado, é necessária a comunicação objetiva, concisa e efetiva entre os membros de todas as equipes de saúde ${ }^{(1)}$. A comunicação caracteriza-se como um dos pilares da qualidade em serviços de saúde e articula mudanças frente às suas fragilidades para o alcance do melhor padrão de desempenho ${ }^{(2)}$.

A comunicação como ferramenta de gestão relacionada à qualidade em saúde possibilita o compartilhamento de informações entre os membros da equipe multiprofissional, previne erros, favorece a transmissão das mensagens de forma segura, além de promover um cuidado direcionado e mais qualificado ${ }^{(2-3)}$. Os programas de treinamento para desenvolver habilidades em comunicação, simulações práticas e proposições para apresentar informações do usuário, constituem formas efetivas de transpor barreiras à comunicação ineficaz e, consequentemente, melhorar os processos de cuidados, a qualidade dos serviços em saúde e a segurança do paciente ${ }^{(4)}$.

A qualidade do cuidado em saúde é um indicador que tem sido incorporado pelas instituições de saúde, para melhorar a prestação do serviço. Esse indicador permite identificar problemas e intervir, buscando solucioná-los. Por isso, configura-se como uma das vertentes que visa garantir o exercício profissional seguro, legal e ético, sem prejuízo para o usuário que é assistido $^{(5)}$.
Em relação à equipe de enfermagem, os registros representam uma ferramenta imprescindível no processo de interlocução do cuidado interprofissional. Eles estabelecem a comunicação entre os profissionais da equipe de enfermagem e os demais integrantes da equipe de saúde, embasando e orientando o planejamento, a prestação e a avaliação do cuidado, assim como qualquer outra ação ou decisão referente à saúde do usuário ${ }^{(6)}$.

Por se tratar de um instrumento legal, os registros de enfermagem devem apresentar as seguintes características: constar de impressos devidamente identificados com dados do usuário, escritos com coerência e coesão, fidedignos, com data e horários específicos, claros, objetivos, com identificação do autor, em ordem cronológica, com letra legível, sem rasuras e contendo todas as informações referentes ao processo de cuidado do usuário, quando no serviço de saúde, tanto no âmbito da atenção primária como na secundária e/ou terciária. Qualquer inverdade, incompletude ou até inexistência de dados e informações, além de gerar implicações éticas e legais, comprometem principalmente a segurança do usuário e a qualidade do cuidado ${ }^{(7)}$.

Este conjunto de informações de saúde pertence ao usuário, cabendo aos profissionais o sigilo da informação e, ao hospital, a guarda segura do prontuário com esses registros. Este orienta todos os profissionais no desenvolvimento do cuidado prestado, sobretudo os que 
compõem a equipe de enfermagem que presta cuidados diretos; favorece a elaboração dos diagnósticos de enfermagem; serve de direcionamento para metas a serem alcançadas e intervenções a serem adotadas, de acordo com o plano de cuidado. Logo, esse instrumento de comunicação deve apresentar as necessidades dos usuários, as condutas clínicas implementadas e ainda os elementos indispensáveis para continuidade e avaliação desse processo ${ }^{(7-8)}$.

A análise de registros em prontuários favorece a investigação, correção e reavaliação do plano terapêutico, além de colaborar com a auditoria, o ensino, a pesquisa e a extensão em saúde. Sendo assim, os registros em prontuários constituem-se em aliados para respaldar a prática de todos os profissionais da equipe de saúde ${ }^{(9)} \mathrm{e}$ contribuem para a segurança do paciente.

Motivadas por essa perspectiva, no interstício de prática hospitalar do componente curricular Gestão e Educação Permanente em Saúde e Enfermagem, do Curso de Enfermagem de uma universidade pública da Bahia, estudantes e docente debruçaram-se sobre essa temática.

Com base no exposto, estudos que discutam o panorama dos registros de enfermagem são relevantes, pois contribuem para o avanço do conhecimento sobre a necessidade e o benefício do atendimento, no mínimo, das normativas éticas legais que embasam registros em prontuários. Além disso, a reflexão sobre o impacto desses, para usuário, serviço e profissionais envolvidos na rede de cuidados, configura-se como uma das metas a serem alcançadas para a segurança do paciente.

Este artigo tem como objetivo relatar uma experiência de Educação Permanente em Saúde (EPS) para o aprimoramento dos registros de enfermagem em prontuários.

\section{Método}

Trata-se de estudo descritivo, de abordagem qualitativa, do tipo relato de experiência, sobre a ação de EPS acerca de registros de enfermagem realizados em unidades de internação de um hospital de ensino público, geral, de grande porte, situado na cidade de Salvador, Bahia,
Brasil. O estudo foi desenvolvido no período de 14 de junho a 19 de julho de 2018, predominantemente no turno matutino das quartas e quintas-feiras.

A ação foi desenvolvida por seis graduandas de enfermagem e uma docente durante a realização de práticas do referido componente curricular, que tem como um dos seus objetivos proporcionar ao discente "reconhecer e aplicar a Educação Permanente em Saúde como instrumento da gestão". Durante essa prática, alocada em um setor sob o acompanhamento direto da enfermeira coordenadora de enfermagem e a supervisão da docente do curso, cada discente desenvolve atividades de gerenciamento. $\mathrm{Na}$ oportunidade, cabe a cada discente identificar e intervir em demandas emergentes dos respectivos setores, desde que guardem relação com, pelo menos, um dos objetivos do componente curricular. A ação pode ser individualizada ou intersetorial. É importante destacar que o problema/demanda prioritariamente deve ser compartilhado e consensuado com os gestores do serviço, tanto quanto factível, e que as discentes tenham governabilidade sobre ele.

Como estratégia para identificar potenciais problemas na gestão de unidades de saúde hospitalar, buscando correlacionar com o Programa Nacional de Segurança do Paciente (PNSP), instituído pela Portaria Ministerial n. 529, de 1 abril de 2013, as discentes escolheram a meta número dois do PNSP, que propõe a melhoria da comunicação entre os profissionais de saúde ${ }^{(10)}$.

O recorte da meta escolhida para abordagem foi direcionado para a comunicação escrita intra e interprofissionais da equipe de enfermagem e demais membros da equipe de saúde.

Objetivando consubstanciar a problemática naquele contexto e ampliar a relevância da intervenção, foram feitos contatos com os coordenadores de cinco unidades de internação, os quais ratificaram a necessidade e o interesse pela proposta.

Após a autorização, as discentes observaram os registros realizados pelos profissionais de enfermagem daquelas cinco unidades, durante nove dias, período compreendido entre a última semana do mês de junho e a primeira semana do mês de julho de 2018. 
Para tanto, foi utilizado roteiro elaborado e adaptado de um estudo quantitativo realizado em $2012^{(11)}$ sobre registros de enfermagem, contendo as seguintes variáveis previstas em registros de prontuários: data, hora, rasuras, espaços em branco, identificação do profissional/categoria, nome completo e registro no Conselho Regional de Enfermagem (Coren), continuidade das informações, erro ortográfico, anotação e evolução de enfermagem, não inclusão de comentário/crítica, letra ilegível, palavras generalizadas/evasivas, abreviaturas não padronizadas e informações não relacionadas ao usuário.

Após a observação de 267 registros de enfermagem, as discentes descreveram as variáveis que mais despertaram a atenção no grupo e analisaram-nas à luz do Código de Ética dos Profissionais de Enfermagem vigente e do Programa Nacional de Segurança do Paciente ${ }^{(10,12)}$. As reflexões advindas dessas experiências balizaram a intervenção junto ao grupo de trabalhadores da enfermagem em três das cinco unidades de internação. Duas coordenadoras não conseguiram compatibilizar os horários das respectivas equipes durante o período das práticas de discentes e docente.

As intervenções ocorreram no formato da estratégia Educação Permanente em Saúde. A Portaria n. 1.996, de 20 de agosto de 2007, fornece as diretrizes para o desenvolvimento da Política de EPS como ferramenta de gestão em saúde, a qual busca fomentar mudanças nas dinâmicas institucionais e fundamenta-se nos conceitos do ensino problematizador e da aprendizagem significativa $^{(13)}$.

Nesse sentido, adota como premissa a vivência real no cotidiano do trabalho, e o ensino-aprendizagem ocorre com base na reflexão daquele contexto de labor. Essa compreensão oportuniza ao trabalhador repensar condutas, procurar novas estratégias e caminhos para a superação de dificuldades individuais e coletivas, relacionadas aos registros de enfermagem nos prontuários.

Desse modo, a intervenção foi feita com base nos problemas enfrentados na realidade, levando em consideração os conhecimentos e as experiências que o trabalhador possui, visando a transformação das práticas profissionais e da própria organização do trabalho, reconhecendo os registros como importante parte desse universo laboral.

Foram realizadas quatro rodas de conversa, uma com os enfermeiros das três unidades, e três reuniões com a equipe de nível técnico; esta última agrupada por unidade de internação.

Participaram 24 enfermeiros, 33 técnicos, 3 auxiliares de enfermagem. As graduandas e a docente conduziram o giro dos quatro momentos, assim distribuídos: dia 10 de julho de 2018, participaram 5 enfermeiros e 11 técnicos em enfermagem. No dia 11 de julho de 2018, a intervenção contou com a presença de 1 enfermeiro, 12 técnicos em enfermagem e 2 auxiliares de enfermagem. Em 13 de julho de 2018, compareceram 10 técnicos em enfermagem e 1 auxiliar de enfermagem. Para finalizar, no dia 17 de julho de 2018, a roda girou com 18 enfermeiros presentes.

Propositadamente, optou-se por reunir um quantitativo de enfermeiros em grupo distinto. Dentre esses, três eram coordenadores de enfermagem e os demais enfermeiros do cuidado direto. Devido à incompatibilidade de horário, seis enfermeiros estiveram presentes nas rodas de conversa iniciais.

A escolha por reunir essa categoria teve o propósito de ampliar a discussão acerca do papel do profissional enfermeiro em relação à supervisão do trabalho de técnicos e auxiliares de enfermagem, incluindo os registros em prontuários.

Quanto ao agrupamento de técnicos e auxiliares por unidade de internação, a intenção foi apresentar resultado conforme o setor, visando despertar o sentimento de responsabilidade pelos registros nos prontuários e, assim, evitar a transferência para o coletivo de outras unidades.

Cada encontro durou, em média, duas horas, tempo considerado suficiente para as considerações das discentes, discussão e reflexão dos trabalhadores acerca dos aspectos envolvidos no processo de trabalho que impactam nos registros de enfermagem. 


\section{Resultados da experiência}

A qualidade do cuidado em saúde necessita de instrumentos para qualificar e ampliar a segurança dos usuários do serviço de saúde, de forma a apontar e corrigir condutas para o melhor desenvolvimento da assistência. Os registros de enfermagem funcionam sob essa ótica, à medida que são utilizados para garantir a cronologia do que sucedeu no tempo em que aquele usuário esteve sob os cuidados de um serviço de saúde específico. É importante ressaltar que os profissionais de enfermagem precisam saber a importância do relato adequado nos registros, pois, além de serem permanentes, eles direcionam a cadeia subsequente do cuidado. Por isso, devem expressar com fidedignidade os cuidados prestados ${ }^{(14-15)}$.

Destarte, as anotações, para serem de qualidade e garantirem o cuidado integral ao sujeito, devem ser organizadas, imparciais, coerentes, integrais, legítimas, adequadas ao contexto, atuais, sem rasuras, legíveis e devem conter a identificação completa do profissional ${ }^{(15)}$.

Nesta experiência, os aspectos que mais se destacaram nas observações dos prontuários referem-se à identificação profissional incompleta e à inconsistência das informações contidas nas anotações e evoluções de enfermagem. Além disso, também se destacaram a ausência da hora no registro, o elevado índice de rasuras e a presença de espaços em branco entre o final do registro e a assinatura do profissional.

Entretanto, a maior gravidade relacionou-se a sete prontuários de usuários internados em uma das unidades observadas, onde não foi encontrado nenhum registro de enfermagem. Vale salientar que, nessa unidade, foi observado o maior conjunto de não conformidades relacionadas aos registros de enfermagem. Como ponto positivo, observou-se que não houve troca de informações relacionadas aos registros de usuários.

O art. 35, do Capítulo II do Código de Ética dos Profissionais de Enfermagem (CEPE), registra que é dever do profissional de enfermagem "Apor nome completo e/ou nome social, ambos legíveis, número e categoria de inscrição no Coren, assinatura ou rubrica nos documentos, quando no exercício profissional", sendo facultativo o uso de carimbo. No entanto, a experiência em apreço revelou predomínio de identificação incompleta, sugerindo que os profissionais de enfermagem precisam ampliar a atenção e o conhecimento quanto a essa responsabilidade ética e legal ${ }^{(12)}$.

Quanto às anotações e evoluções de enfermagem, a experiência revelou fragmentação e pouca aderência às recomendações do Processo de Enfermagem, dada a incipiência ou mesmo inconsistência das informações contidas nos registros.

O histórico de enfermagem foi encontrado na maioria dos prontuários, denotando valorização da história saúde-doença como etapa inicial do processo de cuidado, por parte dos enfermeiros. Também foram encontradas evoluções e alguns planos de alta de enfermagem, porém as etapas de diagnóstico e prescrição de enfermagem não foram localizadas.

Essa fragmentação não contempla os aspectos sistemático e contínuo do Processo de Enfermagem, os quais possibilitam identificar as necessidades e intervenções correlatas, bem como o monitoramento da evolução clínica e ajuste das ações para o alcance da recuperação, estabilização da saúde ou mesmo da finitude do usuário. Essa forma irregular sugere que os enfermeiros priorizam as ações do dia a dia em detrimento da Sistematização da Assistência de Enfermagem (SAE).

A descontinuidade dos conteúdos nas anotações de enfermagem foi uma característica expressiva na maioria dos registros, não possibilitando revelar a evolução clínica dos usuários, do mesmo modo que o trabalho executado pela equipe.

Considerando que esses registros são indicadores de qualidade do cuidado, descrevê-los incorretamente, sem horário e de maneira descontínua são fatores que comprometem a criação de indicadores, avaliação e certificação. Pode até mesmo impedir de servirem como amparo jurídico, seja para os trabalhadores, para o serviço ou para o usuário. 
Registros em prontuários, tanto para os profissionais de enfermagem como para outros da equipe de saúde, constituem-se em uma obrigatoriedade legal. No caso da enfermagem, a Lei do Exercício Profissional, n. 7.498/1986, traz, no seu art. n.14, a incumbência de anotar no prontuário do paciente todas as atividades procedentes da assistência; no CEPE, os art. n. 36 a 38, reforçam o dever de

\begin{abstract}
Registrar no prontuário e em outros documentos, as informações inerentes e indispensáveis ao processo de cuidar de forma clara, objetiva, cronológica, legível, completa e sem rasuras [...] Documentar formalmente as etapas do processo de Enfermagem, em consonância com sua competência legal [...] Prestar informações escritas e/ou verbais, completas e fidedignas, necessárias à continuidade da assistência e segurança do paciente ${ }^{(12)}$.
\end{abstract}

Portanto, fica explicitado que os registros de enfermagem em prontuários, acerca das ações ou atividades junto ao usuário, fazem parte da responsabilidade legal e ética dos profissionais de enfermagem.

Esses aspectos também foram encontrados em estudo realizado em dois hospitais em Maceió (AL) que investigou, no período de maio a dezembro de 2012, por meio da análise do conteúdo dos registros de enfermagem nos prontuários dos pacientes internados, "[...] as contribuições do conteúdo dos registros para a prática assistencial baseada no processo de enfermagem"(17:24). Os resultados da pesquisa evidenciaram que o conteúdo dos registros é deficiente, não retrata a realidade do paciente, nem tampouco a assistência de enfermagem prestada, o que, por sua vez, é um dado preocupante, que se repete em outras pesquisas que envolvem a mesma temática e expõe ética e legalmente as profissionais de enfermagem ${ }^{(17)}$.

Outro estudo, desenvolvido em um hospital de ensino em Minas Gerais, entre fevereiro e março de 2015, descreveu a importância das anotações de enfermagem no prontuário do paciente para a equipe de enfermagem e discutiu as implicações profissionais e institucionais dos registros de enfermagem. O estudo mostrou que grande parte da equipe reconheceu a importância dos registros de enfermagem, todavia desconhecia a legislação e as sanções ético-legais advindas da não conformidade ${ }^{(7)}$.

A análise dos manuscritos em prontuários possibilitou o levantamento de aspectos que propiciaram reflexões acerca das implicações de registros de enfermagem (in)corretos na qualidade do cuidado e na segurança do paciente. Também favoreceu percepções minuciosas quanto à insuficiência de informações que assegurem o cuidado integral e sistematizado de acordo com a SAE.

Apesar de o processo de observação ter se destinado aos registros dos profissionais de enfermagem, inevitavelmente identificaram-se alguns casos de ausência de registros de outras categorias da equipe de saúde. Essa ocorrência dificulta, quando não impede, a comunicação interprofissional e consequentemente fragiliza a segurança do usuário.

Ainda nessa perspectiva, foram observados, nos prontuários, acúmulos de papéis desordenados, o que denota pouca valorização das informações ali contidas. Esse panorama ampara a justificativa de remover os registros "mais antigos", considerados desnecessários para ações, inviabilizando o acesso às informações referentes ao passado próximo e, portanto, impedindo a análise evolutiva da condição de saúde do usuário.

Sendo o prontuário um conjunto de documentos que mostra o histórico de atendimentos de saúde do usuário do serviço de saúde, não acessar a totalidade das informações fragiliza a cadeia de comunicação interprofissional e aumenta a vulnerabilidade às falhas no processo do cuidado. Destaca-se que o prontuário, no campo de prática das discentes, ainda não é eletrônico e os registros manuscritos são feitos em papel. Essas características aumentam a dificuldade de manuseio, arquivo e conservação.

Em todas as quatro rodas de conversa, os participantes inicialmente mostraram-se surpresos com os resultados apresentados. Entretanto, na sequência, muitos reconheceram a deficiência na execução dos registros de enfermagem e associaram-na à falta de atenção, sobrecarga de trabalho decorrente de subdimensionamento de 
pessoal, desconhecimento da legalidade, insuficiência de espaço no prontuário para registro de intervenções realizadas e escassez de tempo para atender às demandas da rotina do setor.

Ao final dos giros das rodas, ficou evidente, para as autoras, que os profissionais entenderam e reconheceram os riscos das inconsistências dos registros em enfermagem. Mostraram-se sensibilizados quanto à possibilidade de os registros valorizarem o trabalho realizado, comprovarem a produtividade, a continuidade e a qualidade do cuidado prestado, implicando diretamente na segurança do paciente, no respaldo ético legal e em aspectos financeiros da saúde.

Apesar das dificuldades relacionadas à execução dos registros corretos, são necessárias iniciativas que estimulem mudanças de comportamento e reflexão crítica acerca de como melhorar a prática dos registros, assim como a supervisão, o acompanhamento e a orientação dos enfermeiros nos casos em que as não conformidades forem evidenciadas.

$\mathrm{Na}$ sequência das rodas de conversa, foram entregues fôlderes às coordenações, com esclarecimentos relativos à temática e ao plano operacional das graduandas, para distribuição entre os profissionais de enfermagem que não participaram da socialização. Desse modo, o conteúdo poderia ser acessado por toda a equipe. Outra estratégia, foi a elaboração de banner com informações acerca das responsabilidades éticas e legais envolvidas nos registros em prontuários e as implicações que registros (in)corretos podem gerar para os usuários, os profissionais e o serviço de saúde.

A experiência confirmou que a EPS, também no contexto hospitalar, propicia aprendizagens significativas e amplia a possibilidade de implementação de mudanças almejadas no serviço ${ }^{(18)}$. Similar ao constatado em outro estudo, a EPS mostrou-se eficaz como estratégia para melhorar a qualidade dos registros de enfermagem, de modo a estimular o pensamento crítico e reflexivo dos profissionais ${ }^{(19)}$.

Diálogos com profissionais de enfermagem sobre a conformidade do registro em prontuário, de acordo as legislações vigentes, é uma ferramenta importante de EPS que pode ser utilizada no hospital.

\section{Conclusão}

Frente à importância dos registros de enfermagem em prontuários, livres de não conformidades, torna-se relevante reorganizar o serviço, de modo que não haja sobrecarga de trabalho e subdimensionamento de pessoal, para que o profissional de enfermagem tenha condições de exercer sua função.

É necessário que os enfermeiros, como lideranças, revisem e supervisionem periodicamente os registros realizados nos prontuários, com o propósito de orientar, esclarecer e reforçar o conhecimento sobre aspectos que são relevantes para concretizar e garantir a segurança do usuário, da equipe e ainda o reconhecimento da organização.

$\mathrm{Na}$ mesma perspectiva, os profissionais de enfermagem devem ser cotidianamente alertados tanto sobre as suas responsabilidades éticas e legais quanto da sua práxis, haja vista tais processos de trabalho terem como centralidade a vida de seres humanos.

A experiência confirmou que a EPS, além de favorecer aprendizagem significativa para as autoras, pôde transformar os processos de trabalho da saúde, por ser uma modalidade de suporte e elemento de apoio para qualificá-lo.

Ter refletido, juntamente com os trabalhadores, sobre registros de enfermagem, reforçou a disposição de aproximar e produzir experiências que fomentem a participação crítica e ativa das autoras na construção de seus conhecimentos.

\section{Colaborações:}

1 - concepção, projeto, análise e interpretação dos dados: Neuranides Santana, Maiara da Silva Brandão Rodrigues, Caren Lorena Menezes Freitas, Rebeca Lopes Oliveira, Danielle Silva dos Santos e Luanda Karina Oliveira de Sousa Barbosa; 
2 - redação do artigo e revisão crítica relevante do conteúdo intelectual: Neuranides Santana, Maiara da Silva Brandão Rodrigues, Caren Lorena Menezes Freitas, Rebeca Lopes Oliveira, Danielle Silva dos Santos e Luanda Karina Oliveira de Sousa Barbosa;

3 - aprovação final da versão a ser publicada: Neuranides Santana.

\section{Referências}

1. Feuerwerker LCM. Micropolítica do Trabalho e o Cuidado em Saúde. Porto Alegre, REDE UNIDA [Internet]. [cited 23 Oct 2019] 2014:35-62. Available from: http://www.saude.sp.gov.br/resources/ humanizacao/biblioteca/dissertacoes-e-teses/ micropolitica_e_saude_laura_camargo.pdf

2. Linch GFDC, Lima AAA, Souza END, Nauderer TM, Paz AA, Costa C. An educational intervention impact on the quality of nursing records. Rev Latino-Am Enfermagem. 2017;25:e2938. DOI: $10.1590 / 1518-8345.1986 .2938$

3. Carneiro SM, Dutra HS, Costa FM, Mendes SE, Arreguy-Sena C. Uso de abreviaturas nos registros de enfermagem em um hospital de ensino. Rev RENE. 2016;17(2):208-16.

4. Nogueira JWS, Rodrigues MCS. Comunicação efetiva no trabalho em equipe em saúde: um desafio para a segurança do paciente. Cogitare enferm. 2015;20(3):636-40.

5. Galhardi NM, Escobar EMA. Indicadores de qualidade de enfermagem. Rev Ciênc Med. 2016;24(2):75-83.

6. Rojahn D, Souza I, Locatelli P, Hermann R, Ascari RA. Comunicação efetiva em registros de enfermagem: uma prática assistencial. UNINGÁ Rev. 2014;19(2):9-13.

7. Borges FFD, Azevedo CT, Amorim TV, Figueiredo MAG, Ribeiro RGM. Importância das anotações de enfermagem segundo a equipe de enfermagem: implicações profissionais e institucionais. Rev enferm Cent-Oeste Min. 2017;7:e1147. DOI: 10.19175/recom.v7i0.1147

8. Morais CGX, Batista EMDS, Castro JFL, Assunção SS, Castro GMO. Registros de enfermagem em prontuário e suas implicações na qualidade assistencial segundo os padrões de acreditação hospitalar: um novo olhar da auditoria. Rev ACRED. 2015;5(9):64-84.
9. Araujo MM, Diniz SOS, Silva OS. Registros de enfermagem: reflexões sobre o cotidiano do cuidar. ABCS Health Sci. 2017;42(3):161-5.

10. Brasil. Ministério da Saúde. Portaria n. 529, de 1 de abril de 2013. Institui o Programa Nacional de Segurança do Paciente. Brasília; 2013.

11. Barral LNM, Ramos LH, Vieira MA, Dias OV, Souza LP. Análise dos registros de enfermagem em prontuários de pacientes em um Hospital de Ensino. Rev Min Enferm. 2012;16(2):188-93.

12. Conselho Federal de Enfermagem. Resolução n. 564, de 6 de novembro de 2017. Aprova o novo Código de Ética dos Profissionais de Enfermagem [Internet]. Brasília (DF); 2017 [cited 2019 Aug 13]. Available from: http://www.cofen.gov.br/ resolucao-cofen-no-5642017_59145.html

13. Brasil. Ministério da Saúde. Gabinete do Ministro. Portaria n. 1.996, de 20 de agosto de 2007. Dispõe sobre as diretrizes para a implementação da Política Nacional de Educação Permanente em Saúde. Brasília (DF); 2007.

14. Borges MD, Silva RDO, Andrade KBS, Pereira SRM, Almeida LF, Mendonça HD. Avaliação dos Registros de Enfermagem acerca das Manifestações Clínicas de Cardiotoxicidade. Rev Enferm Atual In Derme [Internet]. [cited 13 Oct 2019]. 2018;86(24):1-10. Available from: http://revistaenfermagematual. com.br/index.php/revista/article/view/77

15. Rodrigues MS, Baptista IMC. A relevância do correto registro de enfermagem para a auditoria de custos. Trabalho apresentado no XX Encontro Latino Americano de Iniciação Científica. Vale do Paraíba; 2016. Anais [Internet]. Vale do Paraíba; 2016 [cited 13 Oct 2019] Available from: http:// www.inicepg.univap.br/cd/INIC_2016/anais/ arquivos/RE_0398_0885_01.pdf

16. Brasil. Presidência da República Lei n. 7.498, de 26 de junho de 1986. Dispõe sobre a regulamentação do exercício da enfermagem e dá outras providências. Diário Oficial da União. Brasília (DF): 198626 jun; Seção 1:1.

17. Silva TG, Santos RM, Crispim LDMC, Almeida LMWS. Conteúdo dos registros de enfermagem em hospitais: contribuições para o desenvolvimento do processo de enfermagem. Enferm Foco. 2016;7(1):24-7.

18. Flores GE, Oliveira DLL, Zocchie DAA. Educação permanente no contexto hospitalar: a experiência que ressignifica o cuidado em enfermagem. Trab educ saúde. 2016;14(2):487-504. 
Neuranides Santana, Maiara da Silva Brandão Rodrigues, Caren Lorena Menezes Freitas, Rebeca Lopes Oliveira, Danielle Silva dos Santos, Luanda Karina Oliveira de Sousa Barbosa

19. Arnemann CT, Lavich CRP, Terra MG, Mello AL, Raddatz M. Educação em saúde e educação permanente: ações que integram o processo educativo da enfermagem. Rev baiana enferm. 2018;32:e24719. DOI: 10.18471/rbe.v32.24719

20. Jesus MC, Silva VA, Mota RS, Costa JCB, Mendes AS, Oliveira MJ. Repercussões da educação permanente nas práticas assistenciais dos profissionais de enfermagem. Rev baiana enferm. 2019;33:e27555. DOI: 10.18471/rbe.v33.27555

Recebido: 30 de agosto de 2019

Aprovado: 2 de novembro de 2019

Publicado: 9 de março de 2020

A Revista Baiana de Enfermagem utiliza a Licença Creative Commons - Atribuição-NãoComercial 4.0 Internacional. https://creativecommons.org/licenses/by-nc/4.0/ Este artigo é de acesso aberto distribuído sob os termos da Licença Creative Commons (CC BY-NC). Esta licença permite que outros remixem, adaptem e criem a partir do seu trabalho para fins não comerciais. Embora os novos trabalhos tenham de lhe atribuir o devido crédito e não possam ser usados para fins comerciais, os usuários não têm de licenciar esses trabalhos derivados sob os mesmos termos. 